\title{
MONOTHERAPY OR POLYTHERAPY FOR CHILDHOOD EPILEPSIES?
}

Oluwaseun Egunsola ${ }^{1}$, Helen M Sammons ${ }^{1}$ and William P Whitehouse $\mathrm{W}^{2,3}$

1Academic Division of Child Health, University of Nottingham, Derbyshire Children's Hospital, Derby DE22 3DT, UK

2,3 School of Medicine, University of Nottingham and Department of Paediatric Neurology, Nottingham Children's Hospital, Nottingham University Hospitals' NHS Trust, Nottingham NG7 2UH, UK

Corresponding author - William P Whitehouse, School of Medicine, University of Nottingham, E Floor East Block, Queen's Medical Centre, Derby Road, Nottingham NG7 2UH, UK

E-mail address - william.whitehouse@nottingham.ac.uk

Telephone no - 01159249924 ext 63329

Key words: Epilepsy syndromes, antiepileptic drug combinations, young people

Word count: 1615 


\section{Background}

Antiepileptic drugs were frequently used as polytherapy until evidence from a series of studies in the late 1970 s and early 1980s suggested that patients derive as much benefit from monotherapy as polytherapy.[1-3]. Antiepileptic drug polytherapy is increasingly becoming popular again and as much as $30-40 \%$ of prescriptions to children are polytherapy.[4, 5]. The availability of new generation AEDs in the last two decades has encouraged polytherapy. AEDs such as lamotrigine, topiramate, levetiracetam, oxcarbazepine, and zonisamide have been approved for paediatric use and are recommended mostly as adjuncts or as second-line agents.[6] Despite the availability of more AEDs, the prevalence of poorly controlled epilepsies still remains the same. About 30\% of epilepsies are resistant to treatment.[7] Drug resistant epilepsies almost always require polytherapy, but the question of the best treatment approach when an initial monotherapy fails is still debatable.

\section{Rational polytherapy}

Rational polytherapy has been suggested for the treatment of epilepsies. It refers to the use of 2 or more drug combinations with different mechanisms of action. The goal is to achieve synergistic or supra-additive efficacy. A combination regimen is supra-additive when it produces a total effect that is higher than the effects of the sum of individual drugs. Rational polytherapy sometimes aims to attain infra-additive toxicity, such that the component drugs in the polytherapy regimen produce a total toxicity less than the sum of the individual toxicities.[8]

Clinical evidence in support of rational polytherapy for epilepsy is sparse. A 1997 multicentre European study, involving 347 adults, reported synergism between sodium valproate and lamotrigine.[9] Patients given sodium valproate with lamotrigine add-on, had better response rate than those given carbamazepine or phenobarbital with add-on lamotrigine. Another multicentre cohort study in Spain, showed that lacosamide, a sodium channel blocker, was more effective (with a higher seizure freedom rate and clinical response) and better tolerated when combined with a non-sodium channel blocker, rather than with another sodium channel blocker.[10] Neither of these studies however evaluated monotherapy. Synergistic effects however do not always occur when AEDs with different mechanisms of action are combined. Brodie et al, reported that retigabine, a new generation AED which enhances 
potassium channel activity, combined with sodium channel or non-sodium channel AEDs showed similar efficacy and safety.[11]

Rational polytherapy requires a sound knowledge of the mechanisms of action of AEDs. A single AED often has multiple mechanisms of action which makes the choice of appropriate combinations challenging.

\section{Monotherapy or polytherapy in epilepsy management}

In the late 1970s, Reynolds, Shorvon and colleagues conducted a series of studies which showed that AED efficacy was higher when optimum concentrations of monotherapy were administered to treatment naïve patients. These studies highlighted the fact that polytherapy is unnecessary as an initial approach to epilepsy treatment.[2, 3] This shaped the landscape of epilepsy management afterwards. Recent studies have also demonstrated relatively comparable efficacy and safety profiles for monotherapy and polytherapy. A systematic search of databases Medline and EMBASE using search terms: 'monotherapy' and 'polytherapy or add-on or adjunct*' and 'epilepsy or seizure*' yielded 6 studies in which efficacy and safety of AED monotherapy was compared with polytherapy (Table 1). A French multicentre study, that compared substitution of monotherapy and add-on treatment in patients with failed initial monotherapy, did not show any significant difference in seizure freedom at 12 months, $50 \%$ seizure reduction at 2 months, and adverse effect profiles of the two treatment groups.[12] Several other large studies have also reported similar efficacy and safety profiles for substituted monotherapy and add-on therapy after failure of initial monotherapy.[13, 14] None of the six studies showed significant difference in epilepsy control.

When drugs, especially those that share similar metabolic pathways and mechanisms of action are combined, they are likely to interact. In a prospective study, Anderson et al reported a significantly higher risk of adverse drug reactions (ADRs) in children receiving polytherapy than monotherapy.[15] Aggregated safety reports for lamotrigine in children have also shown that the risks of developing most ADRs are lower with monotherapy than polytherapy.[16] Also, supra-additive toxicity is likely with polytherapy involving drugs with similar mechanisms of action. For example, combinations of carbamazepine and oxcarbazepine, or gabapentin and pregabalin, or the use of different benzodiazepines should 
usually be avoided. Combinations where the common adverse effects are similar are probably best avoided, at least in the long term. The risk of neurotoxicity is higher when lamotrigine is added to carbamazepine [17] or when lacosamide is co-prescribed with other sodium channel blockers[18], due to pharmacodynamic interactions. Although phenytoin and phenobarbital have different mechanisms, one a sodium channel blocker and the other a gaba-ergic potentiator, their pharmacokinetics interact in complex ways making their interaction rather unpredictable, this makes it difficult to achieve adequate levels without toxicity. 
Table 1: Treatment outcome with monotherapy versus polytherapy

\begin{tabular}{|c|c|c|c|c|c|c|c|c|}
\hline \multirow[b]{2}{*}{ Reference } & \multirow[b]{2}{*}{ Age(yrs) } & \multirow[b]{2}{*}{ Efficacy outcome } & \multicolumn{3}{|c|}{ Efficacy } & \multicolumn{3}{|c|}{ Adverse reactions } \\
\hline & & & Mono & Poly & $P$ value & Mono & Poly & $P$ value \\
\hline \multirow[t]{2}{*}{ †Semah et al, 2014 [12] } & $18-65$ & Seizure freedom at 2 months & $51 \%$ & $45 \%$ & 0.34 & - & - & - \\
\hline & & $50 \%$ seizure reduction at 6 months & $76 \%$ & $84 \%$ & 0.53 & - & - & - \\
\hline †Millul et al, 2013 [13] & $2-86$ & Treatment failure & $27.2 \%$ & $25 \%$ & Ns & $29.2 \%$ & $26.1 \%$ & Ns \\
\hline †Beghi et al, 2003 [14] & $2-70$ & Retention rate at 12 months & $55 \%$ & $65 \%$ & 0.74 & $51 \%$ & $37 \%$ & 0.07 \\
\hline Deckers et al,2001 [19] & $\geq 18$ & Seizure freedom at 12 months & $86 \%$ & $74 \%$ & - & $22 \%$ & $14 \%$ & 0.15 \\
\hline †Jozwiak et al, 2000 [20] & $12-52$ & $50 \%$ seizure reduction at 7 months & $53 \%$ & $50 \%$ & - & - & - & - \\
\hline †Kwan and Brodie, 2000 [7] & $1-87$ & Seizure freedom & $17 \%$ & $26 \%$ & Ns & $26 \%$ & $12 \%$ & 0.25 \\
\hline
\end{tabular}

+Substituted monotherapy versus add-on therapy, Ns-not significant 


\section{Treatment approach to epilepsy}

The goal of treatment is to achieve full seizure control with minimal toxicity. Therefore, it is important to balance the benefits and risks when choosing AEDs. It is generally agreed that monotherapy should be the initial treatment for newly diagnosed epilepsy in children.[21-23] When one AED does not work, a second drug should be introduced while the child is still receiving the ineffective drug. All changes in therapy, whether adding or withdrawing an AED need to be agreed with the parent and the patient. It is important to consider any possible interactions when introducing the new AED. If seizure control is achieved with the new drug, a gradual withdrawal of the ineffective AED should be attempted. However, withdrawal of the first AED depends on whether it was felt to be partially effective. Also important are its adverse effects and how severe a relapse in the epilepsy would be at the time. For example, in a 16 year old, the period just before important examinations would not be an ideal time to withdraw even a probably ineffective AED.

If the newly added AED is ineffective at maximum tolerated dose (with a serum level at the top of the target range if appropriate) or at a dose well above the maximum recommended dose, one of the AEDs should be slowly withdrawn. A new drug could be introduced either at the same time (placing the child on 3 AEDs temporarily) or after one has been withdrawn.

\section{When polytherapy is inevitable}

The cautious delayed withdrawal of a first ineffective AED can be classed a polytherapy, but is generally viewed as sensible, even by staunch advocates of 'mono-therapy'.

\section{Drug resistant epilepsies}

Polytherapy is inevitable in children with drug resistant epilepsies. The International League Against Epilepsy (ILAE) defines drug resistant epilepsy as: 'failure of adequate trials of two tolerated and appropriately chosen and used AED schedules (whether as monotherapies or

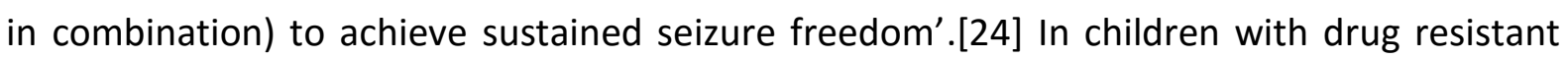
epilepsies, it is common practice to sequentially add AEDs to an existing treatment until seizure control is achieved. However, it is really important to sequentially withdraw ineffective or un-tolerated AEDs, otherwise it is easy to end up with a child on 4 or 5 different AEDs the same time. This increases the likelihood of adverse interactions and more severe 
adverse effects. Sometimes, parents are reluctant to withdraw an AED, but can usually be persuaded by knowing that the drug was ineffective and that the epilepsy will vary through better and worse patches irrespective of what is done with the ineffective AEDs. Furthermore, if the plan leads to a worsening in seizures it can always be reversed.

\section{Electroclinical syndromes}

There are several electroclinical syndromes, some of which are benign and are easily treated or may require no treatment. However, some electroclinical syndromes will almost always require polytherapy.

Infantile Spasms (West syndrome)

Infantile Spasms not due to tuberous sclerosis are generally treated first-line with either hormonal therapy (prednisolone or tetracosactide) or vigabatrin. Vigabatrin is the treatment of choice in children with infantile spasm with tuberous sclerosis. Emerging evidence from a recent multicentre trial reported that hormonal therapy and vigabatrin combination produces faster clinical response and better seizure freedom than hormonal treatment alone. [25]

\section{Dravet syndrome}

Sodium valproate or topiramate is the first-line treatment in children with Dravet syndrome. However, the seizures are often refractory and adjunctive treatment with clobazam and/or stiripentol is usually required.[26] Treatment options are limited because AEDs that target the sodium channel, such as lamotrigine, carbamazepine, oxcarbazepine and phenytoin may aggravate the seizures,[27] and often produce chorea in Dravet syndrome. However some children may in fact benefit from phenytoin.

Lennox-Gastaut syndrome (LGS)

Effective treatment options are few and about $75 \%$ of children with LGS have drug resistant epilepsy.[28] Sodium valproate is often combined with one or two other AEDs, including clobazam, or lamotrigine, or rufinamide, or topiramate.[26] 
CAE usually responds well to treatment with either valproate or ethosuximide, however those with onset under 4 or 5 years of age have a more severe version of CAE,[29] and are typically unresponsive to the usual treatments. However this refractory "Early Onset CAE" can in some cases be well controlled with polytherapy, without adverse effects, even though 3 or 4 AEDs are usually needed, e.g. valproate, ethosuximide, lamotrigine or clobazam.[30]

\section{Conclusion and practical guide}

1. Drug resistant epilepsy is a real challenge and it is easy to over treat with excess doses and combinations.

2. Always have a clear and good reason for using polytherapy.

3. Avoid 3 or more drugs at a time (except during tailing off) in ambulant patients if at all possible.

4. Remember to withdraw an AED when it is ineffective.

5. Ensure that all additions and withdrawals of treatment are agreed to by parents and patient.

6. When seizures increase as the dose is reduced, continue tailing it off, unless you are convinced the patient would be better off on it. Remember epilepsy waxes and wanes unpredictably whatever you do.

7. Discontinue an AED if a serious ADR occurs, record it and avoid the drug next time in that patient.

8. Accept that on rare occasions the general advice given here will need to be modified and individualised for a specific child at a specific time in their illness. 
Contributorship statement: All authors contributed to the writing of this manuscript and approved the final version.

Competing Interests: None

Funding: None 


\section{REFERENCE}

1. Shorvon S, Reynolds E. Unnecessary polypharmacy for epilepsy. Bmj. 1977;1(6077):1635-7.

2. Shorvon S, Chadwick D, Galbraith A, et al. One drug for epilepsy. Bmj. 1978;1(6111):474-6.

3. Reynolds E, Chadwick D, Galbraith A. One drug (phenytoin) in the treatment of epilepsy. The Lancet. 1976;307(7966):923-6.

4. Ackers R, Murray ML, Besag F, et al. Prioritizing children's medicines for research: a pharmacoepidemiological study of antiepileptic drugs. British journal of clinical pharmacology. 2007;63(6):689-97.

5. Hamer HM, Dodel R, Strzelczyk A, et al. Prevalence, utilization, and costs of antiepileptic drugs for epilepsy in Germany-a nationwide population-based study in children and adults. Journal of neurology. 2012;259(11):2376-84.

6. National Institute for Clinical Excellence. Newer drugs for epilepsy in children 2004: Available from: http://www.nice.org.uk/nicemedia/pdf/ta079fullguidance.pdf.

7. Kwan P, Brodie MJ. Early identification of refractory epilepsy. N Engl J Med. 2000;342(5):314-9.

8. Brigo F, Ausserer $\mathrm{H}$, Tezzon $\mathrm{F}$, et al. When one plus one makes three: the quest for rational antiepileptic polytherapy with supraadditive anticonvulsant efficacy. Epilepsy Behav. $2013 ; 27(3): 439-42$.

9. Brodie M, Yuen A, Group S. Lamotrigine substitution study: evidence for synergism with sodium valproate? Epilepsy Res. 1997;26(3):423-32.

10. Villanueva V, López-Gomáriz E, López-Trigo J, et al. Rational polytherapy with lacosamide in clinical practice: results of a Spanish cohort analysis RELACOVA. Epilepsy Behav. 2012;23(3):298-304. 
11. Brodie MJ, French JA, McDonald SA, et al. Adjunctive use of ezogabine/retigabine with either traditional sodium channel blocking antiepileptic drugs (AEDs) or AEDs with other mechanisms of action: evaluation of efficacy and tolerability. Epilepsy Res. 2014;108(5):98994.

12. Semah F, Thomas P, Coulbaut S, et al. Early add-on treatment vs alternative monotherapy in patients with partial epilepsy. Epileptic Disord. 2014;16(2):165-74.

13. Millul A, ludice A, Adami $M$, et al. Alternative monotherapy or add-on therapy in patients with epilepsy whose seizures do not respond to the first monotherapy: An Italian multicenter prospective observational study. Epilepsy Behav. 2013;28(3):494-500.

14. Beghi E, Gatti G, Tonini C, et al. Adjunctive therapy versus alternative monotherapy in patients with partial epilepsy failing on a single drug: a multicentre, randomised, pragmatic controlled trial. Epilepsy Res. 2003;57(1):1-13.

15. Anderson $\mathrm{M}$, Egunsola $\mathrm{O}$, Cherrill J, et al. A prospective study of adverse drug reactions to antiepileptic drugs in children. BMJ open. 2015;5(6):e008298.

16. Egunsola O, Choonara I, Sammons HM. Safety of lamotrigine in paediatrics: a systematic review. BMJ open. 2015;5(6):e007711.

17. Besag FM, Berry DJ, Pool F, et al. Carbamazepine toxicity with lamotrigine: pharmacokinetic or pharmacodynamic interaction? Epilepsia. 1998;39(2):183-7.

18. Novy J, Patsalos PN, Sander JW, et al. Lacosamide neurotoxicity associated with concomitant use of sodium channel-blocking antiepileptic drugs: a pharmacodynamic interaction? Epilepsy Behav. 2011;20(1):20-3.

19. Deckers CL, Hekster YA, Keyser A, et al. Monotherapy versus Polytherapy for Epilepsy: A Multicenter Double-Blind Randomized Study. Epilepsia. 2001;42(11):1387-94.

20. Jozwiak S, Terczynski A. Open study evaluating lamotrigine efficacy and safety in addon treatment and consecutive monotherapy in patients with carbamazepine-or valproateresistant epilepsy. Seizure. 2000;9(7):486-92. 
21. Perucca E, Beghi E, Dulac O, et al. Assessing risk to benefit ratio in antiepileptic drug therapy. Epilepsy Res. 2000;41(2):107-39.

22. Glauser T, Ben-Menachem E, Bourgeois B, et al. ILAE treatment guidelines: evidencebased analysis of antiepileptic drug efficacy and effectiveness as initial monotherapy for epileptic seizures and syndromes. Epilepsia. 2006;47(7):1094-120.

23. Glauser T, Ben-Menachem E, Bourgeois B, et al. Updated ILAE evidence review of antiepileptic drug efficacy and effectiveness as initial monotherapy for epileptic seizures and syndromes. Epilepsia. 2013;54(3):551-63.

24. Kwan P, Arzimanoglou A, Berg AT, et al. Definition of drug resistant epilepsy: consensus proposal by the ad hoc Task Force of the ILAE Commission on Therapeutic Strategies. Epilepsia. 2010;51(6):1069-77.

25. O'Callaghan F, Edwards S, Hancock E, et al. OP50-3017: The International Collaborative Infantile Spasms Study (ICISS) comparing hormonal therapies (prednisolone or tetracosactide depot) and vigabatrin versus hormonal therapies alone in the treatment of infantile spasms: Early clinical outcome. Europ J Paediatr Neurol. 2015;19:S16-S7.

26. The National Institute for Health and Care Excellence. The epilepsies: the diagnosis and management of the epilepsies in adults and children in primary and secondary care2012: Available from: https://www.nice.org.uk/guidance/cg137/chapter/1-Guidance.

27. Dravet C. Dravet syndrome history. Dev Med Child Neurol. 2011;53(s2):1-6.

28. Kluger G, Bauer B. Role of rufinamide in the management of Lennox-Gastaut syndrome (childhood epileptic encephalopathy). Neuropsychiatric disease and treatment. 2007;3(1):3.

29. Roger J, Bureau M, Dravet C, et al. Epileptic Syndromes in Infancy, Childhood and Adolescence. 3rd ed: John Libbey \& Co Ltd 2002. p. 285-303

30. Roger J, Bureau M, Dravet C, et al. Epileptic Syndromes in Infancy, Childhood and Adolescence. 4th ed: John Libbey Eurotext Ltd 2005. p. 315-35. 
